\title{
Bilateral foot drop after COVID-19-related acute respiratory distress syndrome: A case report
}

\author{
Rana Terlemez@, Tuğçe Özekli Mısırlığlu@, Deniz Palamar@, Dilara Okutan@, Kenan Akgün® \\ Department of Physical Medicine and Rehabilitation, Istanbul University-Cerrahpaşa Cerrahpaşa Faculty of Medicine, Istanbul, Turkey \\ Received: January 14, 2021 Accepted: April 02, 2021 Published online: September 01, 2021
}

\begin{abstract}
In these days of the pandemic, we have faced with the locomotor system problems following severe acute respiratory syndrome-coronavirus 2 (SARS-CoV-2) infection. While some of these problems are related to the disease itself, some of them are associated with the prolonged immobilization during the infection. Long-term intensive care unit admissions of patients may also lead to various types of neuropathies, extending the recovery period. The real burden of the novel coronavirus-2019 (COVID-19) is still unclear. In particular, after a prolonged hospitalization period, the duration of rehabilitation may be longer to gain independence in daily living activities. In this report, we present a different aspect of the COVID-19 with bilateral foot drop in a 53-year-old female patient. To the best of our knowledge, this case is the first report of both peroneal and sciatic nerve damage following COVID-19.
\end{abstract}

Keywords: COVID-19, foot drop, sciatic nerve, ultrasound.

Novel coronavirus-2019 (COVID-19) caused by severe acute respiratory syndrome-coronavirus 2 (SARS-CoV-2) infection has rapidly spread all over the world with consequences that are still unpredictable. After the first shock over the acute healthcare systems, the increasing number of COVID-19 survivors are admitted to rehabilitation departments with sequelae. Although the respiratory system is the most frequently involved organ system, additional locomotor system manifestations have been reported in recent studies. ${ }^{[1,2]}$

Foot drop is described as the weakness of the foot and ankle dorsiflexion. Acute unilateral foot drop is a well-known condition, while there is a limited number of data regarding bilateral foot drop. ${ }^{[3]}$ The most common etiology of unilateral foot drop is compression of the deep peroneal nerve, the common peroneal nerve, or the sciatic nerve. Neurological causes of the foot drop also include lumbosacral plexopathy and lumbar radiculopathy. On the other hand, bilateral foot drop is an uncommon condition which may emerge in metabolic diseases such as anorexia nervosa, hypothyroid myopathy, and Chron's disease. Parasagittal intracranial pathologies and caudal equina syndrome may also cause progressive bilateral foot drop. ${ }^{[4]}$ In this report, we present an adult female with bilateral foot drop after severe COVID-19.

\section{CASE REPORT}

A 53-year-old woman presented with distal numbness, weakness, and pain in both legs. She was previously admitted to the hospital with fever, myalgia, and fatigue six months ago. The COVID-19 positivity was confirmed by a reverse transcriptase polymerase chain reaction (RT-PCR) for SARS-CoV-2 through nasopharyngeal swab. Within three days, the patient was hospitalized with the addition of dyspnea. Thoracic computed tomography (CT) revealed bilateral infiltrates, particularly in the lower lobes of both

Corresponding author: Rana Terlemez, MD. İstanbul Üniversitesi-Cerrahpaşa Cerrahpaşa Tıp Fakültesi, Fiziksel Tıp ve Rehabilitasyon Anabilim Dalı, 34098 Fatih, İstanbul, Türkiye. e-mail: ranakaynar@hotmail.com 
lungs. On Day 8, desaturation worsened, and the patient was taken to the intensive care unit (ICU) with acute respiratory distress syndrome (ARDS). Pronesupination cycles were continued until Day 42. After extubation, the patient started to complain about numbness in her lower extremities. Her medical history revealed well-controlled hypertension and previous mastectomy surgery seven years ago with a complete cure for breast cancer. She had no history of smoking. She was overweight with a body mass index of $26 \mathrm{~kg} / \mathrm{m}^{2}$. On physical examination, the scars of pressure ulcers were observed on the zygomatic and sacral bone. She had bilateral distal muscle weakness and hypoesthesia in her lower extremities, most evident on the left side. Muscle strengths for her left ankle dorsiflexion (L4), left great toe extension (L5), and left ankle plantar flexion (S1) were $2 / 5,1 / 5$, and $4 / 5$, respectively, while muscle strengths for her right ankle dorsiflexion (L4), great toe extension (L5), and ankle plantar flexion (S1) were, $4 / 5,2 / 5$, and $5 / 5$, respectively. The sense of touch was reduced in the right L5 and left L4, L5, and S1 dermatomes, and the left Achilles reflex was absent. The clinical impression was suggestive of possible critical illness polyneuropathy, and the patient was referred for electroneuromyography (ENMG). The nerve conduction studies showed normal velocities of the bilateral median and ulnar nerves. The sensory nerve action potential of the left sural was absent, and the right superficial peroneal sensory response was reduced. The right peroneal motor study showed a normal compound muscle action potential at the ankle. However, stimulating above the fibular neck represented a marked reduction in amplitude with slowing of conduction velocity into the demyelinating range. The ENMG studies demonstrated axonal damage to the right peroneal and left sciatic nerve. None of the electrophysiological findings suggested polyneuropathy. Needle ENMG examination demonstrated abnormal spontaneous electromyographic activity with the positive sharp waves and fibrillation potentials with normal motor unit configuration and diminished recruitment in the muscles indicating the left sciatic nerve, including the left short head of the biceps femoris, tibialis anterior, extensor hallucis longus, and medial gastrocnemius. Abnormal spontaneous activity was also found in the right extensor hallucis longus and extensor digitorum brevis muscles. The needle electromyographic

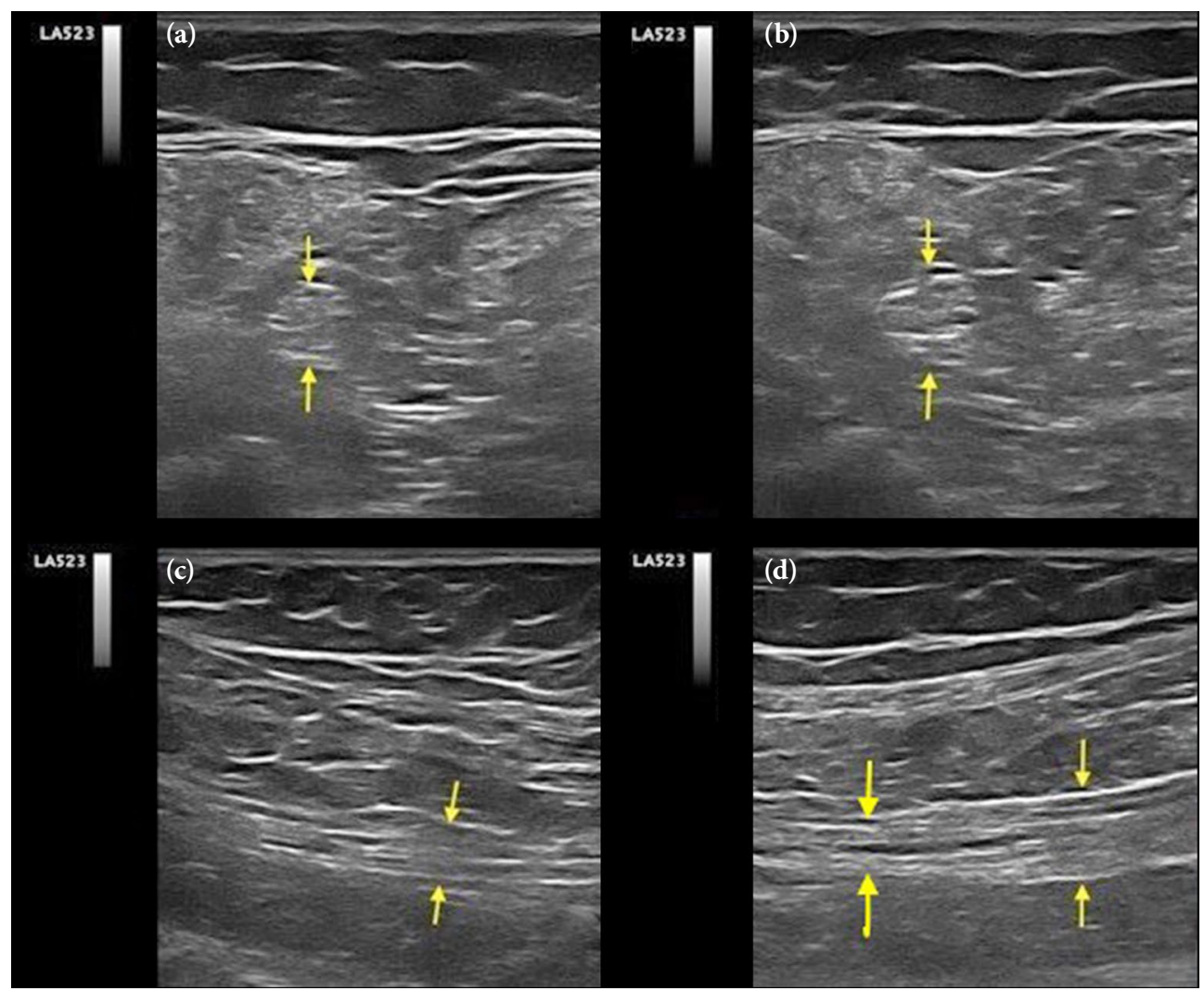

Figure 1. Side-by-side comparison of axial and longitudinal scans of sciatic nerves. (a, c) right side. $(\mathbf{b}, \mathbf{d})$ left side. 
examination of the bilateral gluteus medius, tensor fascia lata, and long head of the biceps femoris muscles were normal. The electrophysiologic findings are consistent with a sciatic neuropathy at or proximal to the takeoff to the short head of the biceps femoris. Ultrasound (US) showed an increased thickness of the left sciatic nerve, compared to the right side, in the mid-thigh level (Figure 1). With a history of prolonged hospitalization in the ICU, pressure-induced nerve palsy was considered for right peroneal and left sciatic nerve, and conservative treatment was initially planned for both sides. In follow-up, improvement in muscle strength and gait pattern were observed. A written informed consent was obtained from the patient.

\section{DISCUSSION}

The COVID-19 pandemic has resulted in an enormous challenge for both healthcare and economics all over the world. Moreover, physiatrists have begun to encounter a new population with disabilities following COVID-19 infection. Besides respiratory illness, these patients may face with cognitive problems, deconditioning, critical illness myopathy and neuropathy, dysphagia, joint contractures, pressure ulcers due to central and peripheral nerve involvement, or prolonged hospitalization only. ${ }^{[5]}$ Observational studies and case reports on SARS-CoV-2 have also shown the potential neurotropic effects of the virus. ${ }^{[1,2]}$ Most reports regarding peripheral nerve involvement in COVID-19 patients have described an increased incidence of critical illness polyneuropathy. Differential diagnosis includes prolonged neuromuscular blockade, vitamin deficiencies, electrolyte disturbances, and drug-related neuromuscular disorders. ${ }^{[6,7]}$ On the other hand, prolonged hospitalization in the ICU setting may lead to additional neuropathies, such as positioning-related nerve entrapments. Virus-induced hyperinflammation and hypercoagulability also increase the vulnerability of peripheral nerves. ${ }^{[7,8]}$

The ICUs have played a pivotal role in the fight against COVID-19 during the pandemic. A recent meta-analysis showed that approximately one-third of patients were admitted to ICUs with severe SARS-CoV-2 infection. ${ }^{[8]}$ In the management of COVID-19 patients with ARDS, the prone position is frequently used to improve oxygenation and survival, as recommended by the World Health Organization (WHO). ${ }^{[9]}$ However, patients are not only left in the prone position, but are followed in cycles by lying in the supine and prone position. Although peripheral nerve entrapment is a well-known position-related complication, there are insufficient data on the impact of prone position on peripheral nerves, particularly in ARDS. Bellinghausen et al. ${ }^{[10]}$ and Marinelli et al. ${ }^{[1]}$ reported meralgia paresthetica in COVID-19 patients after prolonged prone position. Malik et al. ${ }^{[12]}$ also reported peripheral nerve injuries involving the ulnar nerve, radial nerve, brachial plexus, median nerve, and sciatic nerve, associated with prone positioning in ARDS patients. To the best of our knowledge, this is the first report of both peroneal and sciatic nerve damage after prolonged hospitalization following COVID-19. The entrapment neuropathies in our patient seemed to be more related to the prolonged supine position. She had scars of pressure ulcers on the zygomatic and the left side of the sacral bone, indicating prolonged positioning for both prone and supine.

Peroneal nerve palsy is the most frequently observed compression neuropathy of the lower extremity. ${ }^{[13]}$ We frequently observe patients with unilateral peroneal nerve entrapment in our daily practice. External compression of the peroneal nerve, usually followed by anesthesia, prolonged hospitalization, casting, tight bracing, compression wrapping, and pneumatic compression device use, often causes peroneal nerve entrapment. Sciatic neuropathy is a rare mononeuropathy, which accounts for $1 \%$ of all patients referred for ENMG. ${ }^{[14]}$ The hip region is a common site of sciatic neuropathy, and the masses and trauma are the main reasons. ${ }^{[15]}$ The causes of sciatic neuropathy in the thigh include external compression during prolonged supine position and posterior thigh compartment syndrome. Distad and Weiss ${ }^{[16]}$ reported that the sciatic nerve entrapment usually occurred at the mid-thigh level in patients with coma. In our US examination, we also found the sciatic nerve was more swollen at the thigh level. Prolonged sitting position on a toilet or total hip arthroplasty have been defined as the causes of sciatic neuropathy. Sciatic nerve injury may also occur at the thigh as a sequela of a penetrating injury. ${ }^{[14,15]}$

In conclusion, careful clinical examination and electrophysiological studies are crucial for the diagnosis of intensive care unit-related entrapment neuropathies. To minimize complications, nursing personnel should be cautious about the correct positioning of the patients. At this stage of the pandemic, we believe that it is of utmost importance to highlight the complications occurring during the intensive care unit stay, which we may face more commonly in the near future. Clinicians should be aware of the increased vulnerability of the peripheral nerves in severe COVID-19. 


\section{Declaration of conflicting interests}

The authors declared no conflicts of interest with respect to the authorship and/or publication of this article.

\section{Funding}

The authors received no financial support for the research and/or authorship of this article.

\section{REFERENCES}

1. Wang D, Hu B, Hu C, Zhu F, Liu X, Zhang J, et al. Clinical characteristics of 138 hospitalized patients with 2019 novel coronavirus-infected pneumonia in Wuhan, China. JAMA 2020;323:1061-9.

2. Mao L, Jin $\mathrm{H}$, Wang $\mathrm{M}, \mathrm{Hu} \mathrm{Y}$, Chen $\mathrm{S}, \mathrm{He} \mathrm{Q}$, et al. Neurologic manifestations of hospitalized patients with coronavirus disease 2019 in Wuhan, China. JAMA Neurol 2020;77:683-90.

3. Stewart JD. Foot drop: Where, why and what to do? Pract Neurol 2008;8:158-69.

4. Shapiro BE, Preston DC. Entrapment and compressive neuropathies. Med Clin North Am 2009;93:285-315.

5. Carda S, Invernizzi M, Bavikatte G, Bensmaïl D, Bianchi F, Deltombe T, et al. COVID-19 pandemic. What should Physical and Rehabilitation Medicine specialists do? A clinician's perspective. Eur J Phys Rehabil Med 2020;56:515-24.

6. Algahtani H, Subahi A, Shirah B. Neurological complications of Middle East respiratory syndrome coronavirus: A report of two cases and review of the literature. Case Rep Neurol Med 2016;2016:3502683.

7. Tsai LK, Hsieh ST, Chao CC, Chen YC, Lin YH, Chang SC, et al. Neuromuscular disorders in severe acute respiratory syndrome. Arch Neurol 2004;61:1669-73.
8. Koralnik IJ, Tyler KL. COVID-19: A global threat to the nervous system. Ann Neurol 2020;88:1-11.

9. Taboada M, González M, Álvarez A, González I, García J, Eiras $M$, et al. Effectiveness of prone positioning in nonintubated Intensive Care Unit patients with moderate to severe Acute Respiratory Distress Syndrome by coronavirus disease 2019. Anesth Analg 2021;132:25-30.

10. Bellinghausen AL, LaBuzetta JN, Chu F, Novelli F, Rodelo AR, Owens RL. Lessons from an ICU recovery clinic: Two cases of meralgia paresthetica after prone positioning to treat COVID-19-associated ARDS and modification of unit practices. Crit Care 2020;24:580.

11. Marinelli L, Mori L, Avanti C, Cotellessa F, Fabbri S, Schenone C, et al. Meralgia paraesthetica after prone position ventilation in a patient with COVID-19. Eur J Case Rep Intern Med 2020;7:002039.

12. Malik GR, Wolfe AR, Soriano R, Rydberg L, Wolfe LF, Deshmukh $S$, et al. Injury-prone: Peripheral nerve injuries associated with prone positioning for COVID-19related acute respiratory distress syndrome. Br J Anaesth 2020;125:e478-e480.

13. Poage C, Roth C, Scott B. Peroneal nerve palsy: Evaluation and management. J Am Acad Orthop Surg 2016;24:1-10.

14. Yuen EC, So YT, Olney RK. The electrophysiologic features of sciatic neuropathy in 100 patients. Muscle Nerve 1995;18:414-20.

15. Yuen EC, Olney RK, So YT. Sciatic neuropathy: Clinical and prognostic features in 73 patients. Neurology 1994;44:1669-74.

16. Distad BJ, Weiss MD. Clinical and electrodiagnostic features of sciatic neuropathies. Phys Med Rehabil Clin N Am 2013;24:107-20. 